\title{
Polyamide capsules via soft templating with oil drops-1. Morphological studies of the capsule wall
}

\author{
Hisham Essawy $•$ Klaus Tauer
}

Received: 27 August 2009 /Revised: 2 December 2009 / Accepted: 4 December 2009/Published online: 6 January 2010

(C) The Author(s) 2009. This article is published with open access at Springerlink.com

\begin{abstract}
Poly(terephthalamide) microcapsules can be reproducibly and easily prepared by interfacial polycondensation around emulsion droplets in water. Oil drops of cyclohexane/chloroform mixture stabilized with poly(vinyl alcohol) containing terephthaloylchloride serve as soft template. The interfacial polycondensation starts immediately after addition of an amine mixture (hexamethylenediamine/diethylenetriamine). Light and scanning electron microscopy prove the formation of capsules with size distribution in the range from a few up to $100 \mu \mathrm{m}$ depending on particular composition of the reaction mixture. The morphology of the capsule wall is characterized by precipitated particles. If instead of pure organic solvents a reactive oil phase is used as template, the capsules can serve in subsequent reactions as templates for the synthesis of composite particles. In this way, styrene can be radically polymerized inside the capsule leading to composite capsules. The capsule morphology is determined by the partition of all components between all phases.
\end{abstract}

Keywords Polyamide capsules · Capsule wall morphology . Radical styrene polymerization $\cdot$ Microreactors

H. Essawy $\cdot$ K. Tauer $(\bowtie)$

Max Planck Institute of Colloids and Interfaces,

Am Mühlenberg 1,

14476 Golm, Germany

e-mail: klaus.tauer@mpikg.mpg.de

H. Essawy

Polymers \& Pigments Department, National Research Center,

Dokki,

12311 Cairo, Egypt

\section{Introduction}

Microcapsules of sizes below $100 \mu \mathrm{m}$, either empty or filled, are of commercial interest for a variety of applications (such as controlled release of fragrances and drugs, nutrient retention, and cosmetic applications) for more than 20 years and also of long lasting scientific interest; see for instance [1-3] and references therein. Exciting examples are the encapsulation of phase change materials for storage of thermal energy [4-6] and the encapsulation of UV absorbers in silica microcapsules for sunscreen applications [7]. Reactive microcapsules that can be applied as selfhealing materials are another spectacular application $[1,8$, 9]. All the particular applications require specific properties of the capsule wall.

The principles of microcapsule formation via an aqueous dispersion as precursor are extensively described in the open literature $[10,11]$. Interfacial polycondensation is a straightforward and frequently applied method to produce suitable microcapsules and has been developed to a versatile technique with a high degree of freedom and controllability of product properties $[12,13]$. The relationship between the properties of the oil phase and those of the polymer forming the capsule wall has been intensely studied because it is important for controlling shape, microstructure, permeability, and mechanical properties of the membrane $[3,13,14]$. A clever approach to control capsule properties by a combination of interfacial polymerization and free radical polymerization was developed by Mahabadi et al. [2, 15, 16]. The authors used template oil droplets, stabilized by poly(vinyl alcohol) (PVAl), that contain besides the hydrophobic isocyanate also hydrophobic monomer(s) and initiator necessary for the radical polymerization following the primary capsule formation via polycondensation. The inter- 
facial polycondensation is started with the addition of an aqueous solution of a hydrophilic diamine. After a certain period of time that is necessary for the formation of the polyurethane capsule wall, the temperature is increased in order to start the radical polymerization. The authors intensely investigated the emulsification process, that is, the dependence of the drop size on the stirrer speed and emulsification time. Furthermore, the authors noticed that, especially at low PVAl concentrations, the stability of the emulsion droplets is insufficient as the concentration of PVAl that is available for the stabilization is reduced due to a side reaction of hydroxyl groups with isocyanate groups. Unfortunately, the authors did not present data (neither electron microscopy nor light microscopy) describing the morphology of the polyurethane membrane as well as the morphology of the final composite particles.

The capsule formation itself can be considered as soft templating of emulsion droplets that is believed to proceed via three steps [10]. The whole process starts with the formation of oligo-polyamides (initial polycondensation reaction). These oligomers form during the second step the primary membrane at the interface between the emulsion droplets and water. The third step is the growth of the membrane. Of course, this is only a very rough and general description that says nothing about the morphology of the membrane. Moreover, the mechanism of the capsule wall formation process is much more complicated because of the action of concentration gradients, partition coefficients, and mass transfer processes. Additionally, the volume ratio of the two phases; the chemical nature of all ingredients (stabilizer and other recipe components; template phase), the hydrodynamic conditions, the polycondensation rate, and solvency of both phases for the monomers and the resulting capsule wall polymer influence the process. The latter point is of special meaning as the stoichiometry of the monomers in the reaction zone is important for the mechanical properties of the capsule wall polymer which must be per se insoluble in both phases. The required insolubility of the capsule wall material in both the continuous and the dispersed phase causes special attention of the experimenter because the morphology of the capsule wall influences the final capsule properties to a great deal. Very probably, the chain ends of the initially precipitated polymer consist of both kinds of reactive groups. Such a situation enables further reaction of the precipitated chains with monomers but also with other chains possessing complementary chain ends. This kind of reactive aggregation of the unstable colloidal particles should lead to a very special structure. A morphology consisting of colloidal particles that are connected with polyamide chains might be the result. Stoichiometric composition must not be given in any volume element of the reaction mixture so that the kinetic processes occur far from equilibrium [17].
Here, we present results using a similar approach as Mahabadi et al. [2, 15, 16], but instead of polyurethane, the primary capsule wall material is polyamide. The oil phase is a mixture of cyclohexane and chloroform that is frequently used for precipitation of polyamide [13, 18]. It is assumed that chloroform might to some extent be able to swell polyamide, whereas cyclohexane is neither able to dissolve nor swell the polymer $[19,20]$.

The wall thickness requirements greatly depend on the application of the capsules. So, studies to what extent and by which means the thickness of the capsules wall can be controlled are of considerable interest. Microscopic studies on the morphology of polyamide membranes and capsule walls are well known [13, 18, 20-26]. However, the discussion of the morphology is restricted to the appearance of both sides of the membrane or the whole capsules in the high-micrometer-size range. Additionally, the thickness of the membrane is typically a few micrometers except in [13] where the membrane thickness is about $0.5 \mu \mathrm{m}$.

The focus of this contribution is on the morphology of the capsule wall because the chemistry of polyamide formation and the mechanism of the interfacial polycondensation are well understood [18, 22-24, 27, 28]. In order to study details of the morphology of the capsule wall, the thickness was minimized. Capsules with thin walls can completely collapse in the dried state leading to a typical wrinkle pattern on microscopy images. These capsules might be useless for applications but useful for studies of the early stage of the development of the membrane morphology. During further reaction, the membrane itself causes changes in the growth conditions because it hinders more and more the encounter of the reaction partners until the polycondensation eventually stops completely [18, 22]. In this study, an attempt was made to control the thickness of the capsule wall in two ways. The first strategy was via the concentration of terephthaloyl dichloride and hexamethylenediamine in the organic phase and in water, respectively, at a given stoichiometric ratio. The second attempt was to carry out after the polycondensation a radical polymerization of styrene inside the capsules.

Particularly, the morphology of the primary capsules and that of the composite particles with polystyrene is described based on light and electron microscopy investigations. These studies reveal surprising results as the morphology of both the polyamide capsule and the polyamide-polystyrene composite is the result of coagulation processes of mainly spherical primary particles. Here, the formation of polytherephthalamide-polystyrene composite capsules is only briefly touched showing the proof of principle. However, the first results were interesting enough to initiate more detailed studies which will be the focus of subsequent reports. 


\section{Experimental}

\section{Materials}

Chloroform (Merck, Darmstadt, Germany), tetrahydrofuran (BDH Prolab), diethylenetriamine (DETA, from Acros 98.5\%), terephthaloyl dichloride (TPC, from Acros 99\%), hexamethylenediamine (HMDA, from Sigma-Aldrich 98\%), cyclohexane (Sigma-Aldrich), poly(vinyl alcohol) (a gift from Wacker, type M05/140 with a content of $\mathrm{OH}-$ groups between 86 and $89 \mathrm{~mol} \%$ ), 2,2'-azobisisobutyronitrile (AIBN from Wako), and styrene (Sigma-Aldrich 99\%) were all used as received except styrene and AIBN. Styrene was distilled under reduced pressure and AIBN was recrystallized from methanol before use. The water was taken from a Seral purification system (PURELAB Plus) with a conductivity of $0.06 \mu \mathrm{S} \mathrm{cm}^{-1}$.

\section{Methods}

\section{Synthesis of poly(terephthalamide) microcapsules (standard procedure)}

Fifteen milliliters of the organic phase (cyclohexanechloroform mixture, 4:1 volume ratio) containing $1 \mathrm{mmol}$ of terephthaloyl dichloride was pre-emulsified into $75 \mathrm{ml}$ of distilled water containing $1 \mathrm{wt} \%$ of PVAl $(750 \mathrm{mg})$ in a jacketed four-neck flask operated by a mechanical stirrer with a six-blade propeller at a stirring rate of $200 \mathrm{rpm}$ for $5 \mathrm{~min}$. Then the stirring speed was increased to $1,200 \mathrm{rpm}$ for $10 \mathrm{~min}$ to intensify emulsification. Thereafter, the stirrer speed was reduced to $400 \mathrm{rpm}$ and $75 \mathrm{ml}$ of distilled water containing $1 \mathrm{wt} . \%$ of PVAl, $1 \mathrm{mmol}$ of HMDA, and $1 \mathrm{mmol}$ of DETA were added within one shot (within about $1 \mathrm{~min}$ ) to start the interfacial polycondensation. The reaction was allowed to complete for $1 \mathrm{~h}$. The excess amine is necessary in order to catch the liberated hydrochloric acid according to a procedure described in [20].

\section{Synthesis of poly(terephthalamide)-poly(styrene) composite particles}

Styrene was part of the oil phase containing $1 \mathrm{mmol}$ terephthaloyl chloride and additionally $1.25 \mathrm{wt} . \%$ of AIBN (relative to the amount of styrene). After completion of the polycondensation, an aliquot of the reaction mixture was removed for comparison analysis before the reactor temperature was increased to $75^{\circ} \mathrm{C}$ to start the radical polymerization of styrene. The reaction was continued for $5 \mathrm{~h}$. After this period of time, styrene was completely polymerized as indicated by the benzaldehyde smell. The capsules/particles were separated from the milky colloidal dispersion by several cycles of centrifugation followed by redispersion while replacing the supernatant with distilled water.

\section{Characterizations}

The capsules/particles were examined regarding size and morphology with optical microscopy (Keyence Corporation, Osaka, Japan) either with an objective VH-Z100 or VH-Z500 allowing magnifications up to 1,000- and 5,000fold, respectively, or scanning electron microscopy (LEO1550 Gemini operating at $3 \mathrm{kV}$ ). Fourier transform infrared spectra were collected on a Varian 1000 spectrophotometer and used to check the composition of the capsules (data not shown). Only the polyamide capsules present in the creamed phase were characterized with microscopy techniques. Dynamic light scattering of the supernatant latex phase, in the case of the composite capsules, was carried out with a NICOMP particle sizer model 370 (Santa Barbara, USA).

\section{Results}

The process of capsule formation as employed here is actually an encapsulation of emulsion droplets via interfacial polycondensation in water as continuous phase. An advantage of using emulsion droplets as soft templates is that, consecutively after or simultaneously with capsule formation, other reactions can be easily carried out. This contribution deals with the consecutively started radical polymerization of styrene in a one-pot procedure by changing the temperature after the formation of the primary capsules.

\section{Polyamide capsules}

Before studying the formation of composite particles, the formation of the primary capsule, especially the morphology of the primary capsule wall, was investigated. Entry 2 in Table 1 represents the standard procedure that has been repeated three times in order to check the reproducibility. The procedure as detailed in the Experimental section is quite robust and reproducible.

It leads to almost only capsules with merely negligible amount of solid particles. This is proven by the photographs of Fig. 1. Panel b of Fig. 1 shows almost complete creaming of the capsules at the end of the polycondensation (cf. Fig. 1) and only minor turbidity of the aqueous phase. Creaming is expected as the density of the oil phase is below that of water. Formally, it is $0.919 \mathrm{~g} / \mathrm{ml}$ (density of cyclohexane and chloroform is 0.779 and $1.8 \mathrm{~g} / \mathrm{ml}$ at $25^{\circ} \mathrm{C}$, respectively) but it will be very likely lower as chloroform has a much higher solubility in water $\left(0.8 \mathrm{~g} / 100 \mathrm{ml}\right.$ at $\left.20^{\circ} \mathrm{C}\right)$ than cyclohexane. 
Table 1 Variation of the monomer concentration and cross-linker content during the formation of the polyamide capsules

\begin{tabular}{|c|c|c|c|}
\hline Entry & Oil phase $(15 \mathrm{ml})$ & Shell component 1 & Shell component 2 \\
\hline 1 & (Cyclohexane-chloroform) & $0.5 \mathrm{mmol} \mathrm{TPC}$ & 0.5 mmol HMDA +0.5 mmol DETA \\
\hline 2 & (Cyclohexane-chloroform) & $1 \mathrm{mmol} \mathrm{TPC}$ & 1 mmol HMDA+1 mmol DETA \\
\hline 3 & (Cyclohexane-chloroform) & $2 \mathrm{mmol} \mathrm{TPC}$ & 2 mmol HMDA +2 mmol DETA \\
\hline 4 & (Cyclohexane-chloroform) & $3 \mathrm{mmol} \mathrm{TPC}$ & 3 mmol HMDA +3 mmol DETA \\
\hline 5 & (Cyclohexane-chloroform) & $1 \mathrm{mmol} \mathrm{TPC}$ & 2 mmol HMDA \\
\hline 6 & (Cyclohexane-chloroform) & $1 \mathrm{mmol} \mathrm{TPC}$ & 1.5 mmol HMDA +0.5 mmol DETA \\
\hline 2 & (Cyclohexane-chloroform) & $1 \mathrm{mmol} \mathrm{TPC}$ & 1 mmol HMDA + 1 mmol DETA \\
\hline 7 & (Cyclohexane-chloroform) & $1 \mathrm{mmol} \mathrm{TPC}$ & 0.5 mmol HMDA +1.5 mmol DETA \\
\hline 8 & (Cyclohexane-chloroform) & $1 \mathrm{mmol} \mathrm{TPC}$ & 2 mmol DETA \\
\hline
\end{tabular}

The composition of the oil phase is constant with a cyclohexane-to-chloroform volume ratio of 4:1; the shell component 1 (TPC) is dissolved in the oil phase; polycondensation is started by addition of an aqueous solution $(75 \mathrm{ml})$ of shell component 2 (amine mixture, i.e., diamine and cross-linker)

Moreover, as the reaction system is not closed and the boiling point of chloroform is about $20^{\circ} \mathrm{C}$ lower than that of cyclohexane, there is a good chance that at least a part of the chloroform evaporates in the course of the overall reaction.

The final polyamide capsules cream much faster than the starting emulsion. Besides the above argumentation regarding the density and the solubility in water, there is another point that must be considered. With the start of the polycondensation reaction, the continuous phase of the emulsion is diluted by a factor of two. This can cause redistribution of the PVAl stabilizer and increasing droplet sizes leading to subsequently faster creaming.

The capsules were characterized with light microscopy (1) immediately after preparation as dispersion still containing at least a part of the oil phase and (2) after drying on the microscopy glass slides (Fig. 2).

Expectedly, the capsules in the wet state possess almost a spherical shape (cf. Fig. 2a, c). Particularly, panel c shows free-floating capsules in very different states of evaporation of the oil phase. But still, all capsules exhibit roundly shaped interfaces to the continuous phase showing some flexibility of the capsule wall allowing minimization of the surface free energy. On the contrary, in the dried state, angled structures with quite a lot of wrinkles can be recognized (cf. Fig. 2b, d). The size distribution of the capsules is quite broad and ranges (in particular panel b) from below 45 to above $75 \mu \mathrm{m}$.

Figure 3 shows scanning electron microscopy (SEM) images of different magnifications of microcapsules repeatedly prepared under standard conditions. The capsules possess an overall size distribution between about 19 and $75 \mu \mathrm{m}$ (cf. Fig. 6b). The general reproducibility of capsule formation process is illustrated by comparing the images of Figs. 2 and 3 displaying capsules of different repeats.

Also, the SEM images (Fig. 3) prove the flexibility of the capsule wall as there is quite a high number of wrinkles per capsule with a lot of U-turns. Furthermore, one should notice that both the number and the arrangement of wrinkles significantly differ from capsule to capsule.

Another morphological level is accessible for investigation with still larger magnification of the SEM images. Increasing the magnification reveals that the primary capsule wall is not homogeneous or smooth but rather heterogeneous and porous (Fig. 4).

The SEM images of Fig. 4 reveal that the capsule wall is apparently a coagulation structure made of almost spherical polyamide particles with an average size below $100 \mathrm{~nm}$.

Fig. 1 Photographs showing the template oil-in-water emulsion after the emulsification (a) and the final capsule dispersion after the polycondensation (b); standard conditions (entry 2 of Table 1); stirring is stopped in both cases; creaming after the emulsification takes place within a few hours but it is much faster after the polycondensation
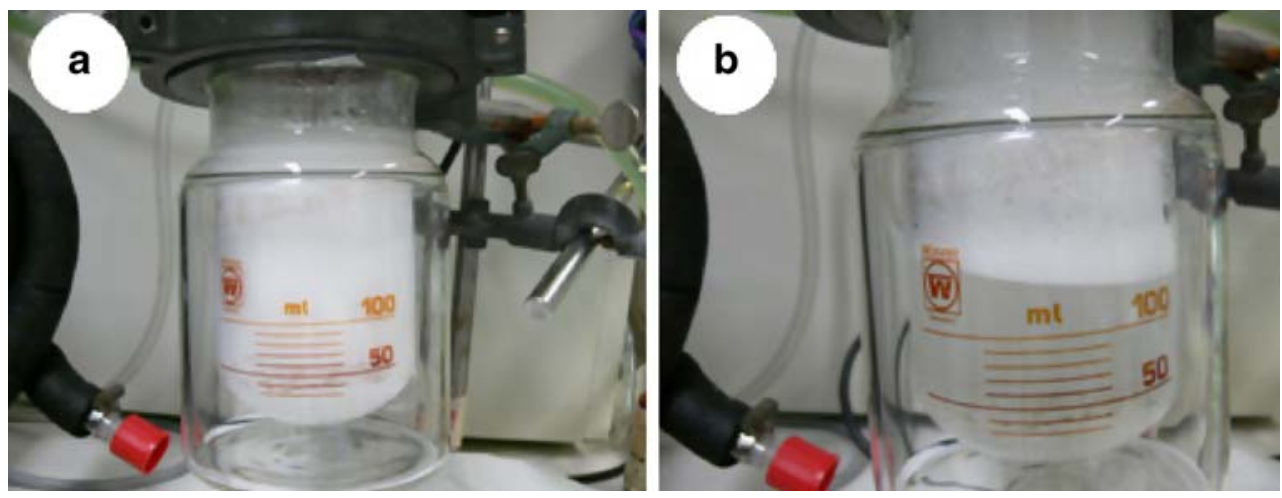
Fig. 2 Light microscopy images of polyamide microcapsules (standard conditions, entry 2 of Table 1) in the dispersion state $(\mathbf{a}, \mathbf{c})$ and in the dried state $(\mathbf{b}, \mathbf{d})$; drying on the microscopy glass slide at ambient conditions; the bar indicates $50 \mu \mathrm{m}$ for (a), (b), and (c) and $25 \mu \mathrm{m}$ for (d)
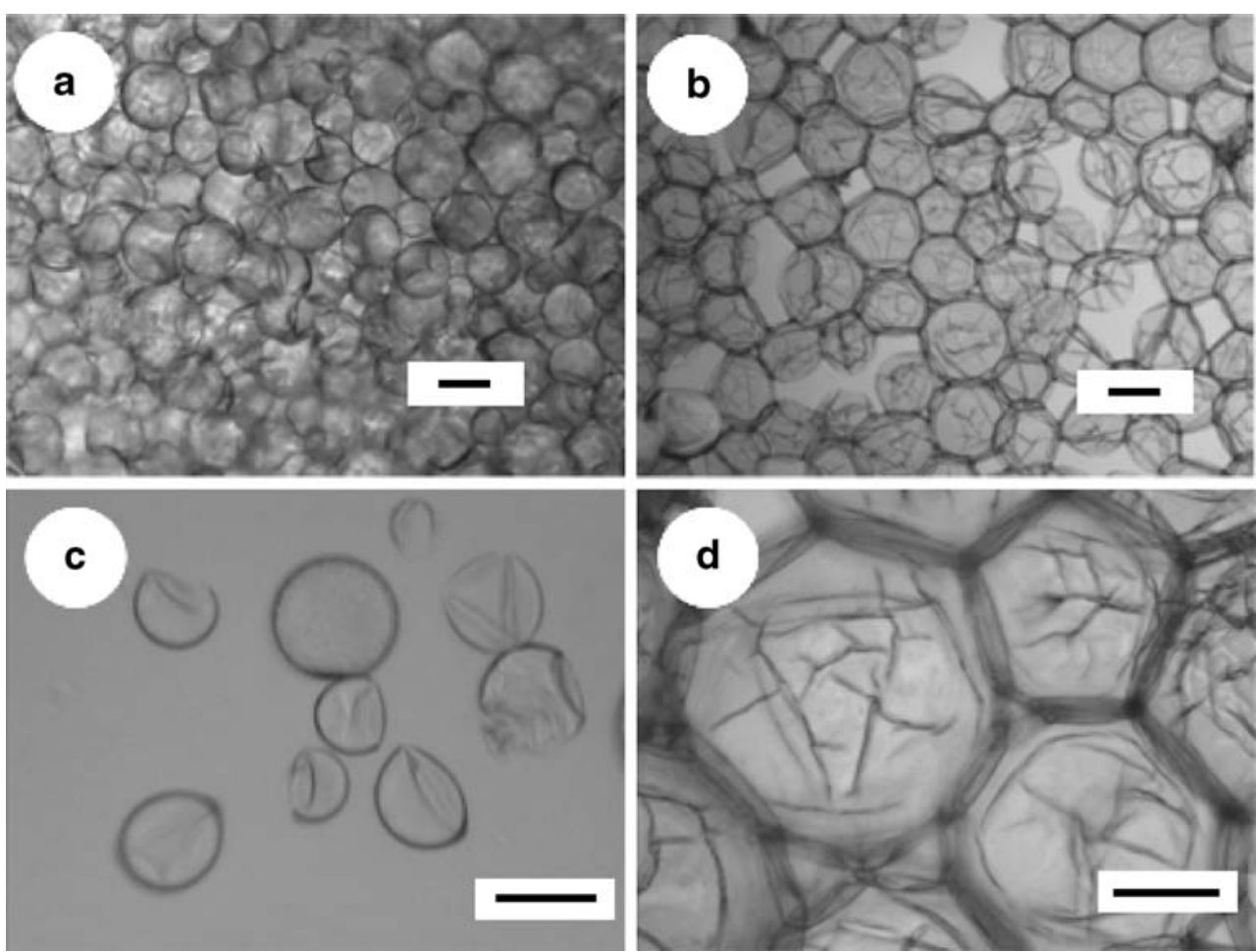

An enlargement of panel d shown in Fig. 5 reveals further morphological details. The polyamide particles arrange in the form of strings as indicated by black lines. These strings consist of a few particles and can be bended if required by the curvature of the capsule wall.

In a series of experiments, the influence of the amount of both shell components on the encapsulation process was studied (entries 1-4 of Table 1) while keeping the amount of oil and water unchanged. It should be mentioned that there is no simple direct relation between the overall amount of monomers and both the capsule size and capsule thickness. The interfacial polycondensation is gradually more hindered with increasing conversion and shell thickness, respectively $[18,22]$. As a result, a gradient regarding composition and molecular weight of the polyamide is developing between the oil and the water
Fig. 3 Scanning electron microscopy images of polyamide microcapsules (standard conditions, entry 2 of Table 1) at different magnifications; the bar indicates $100 \mu \mathrm{m}$ for (a), $20 \mu \mathrm{m}$ for $(\mathbf{b})$, and $10 \mu \mathrm{m}$ for $(\mathbf{c})$ and $(\mathbf{d})$
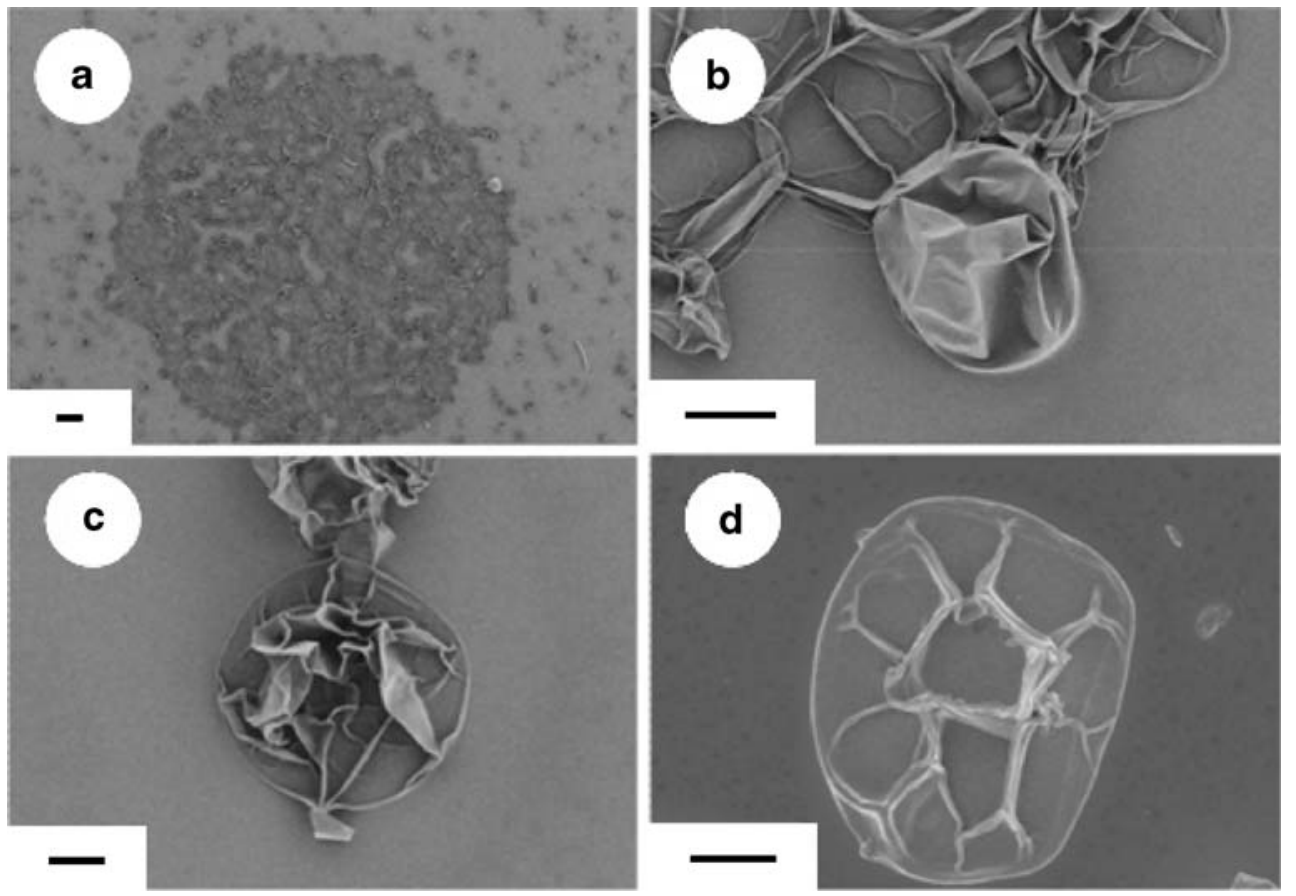
Fig. 4 Scanning electron microscopy images of polyamide microcapsules (standard conditions, entry 2 of Table 1 ) at different magnifications; the bar indicates $3 \mu \mathrm{m}$ for (a), $1 \mu \mathrm{m}$ for (b), $200 \mathrm{~nm}$ for (c), and $100 \mathrm{~nm}$ for $(\mathbf{d})$
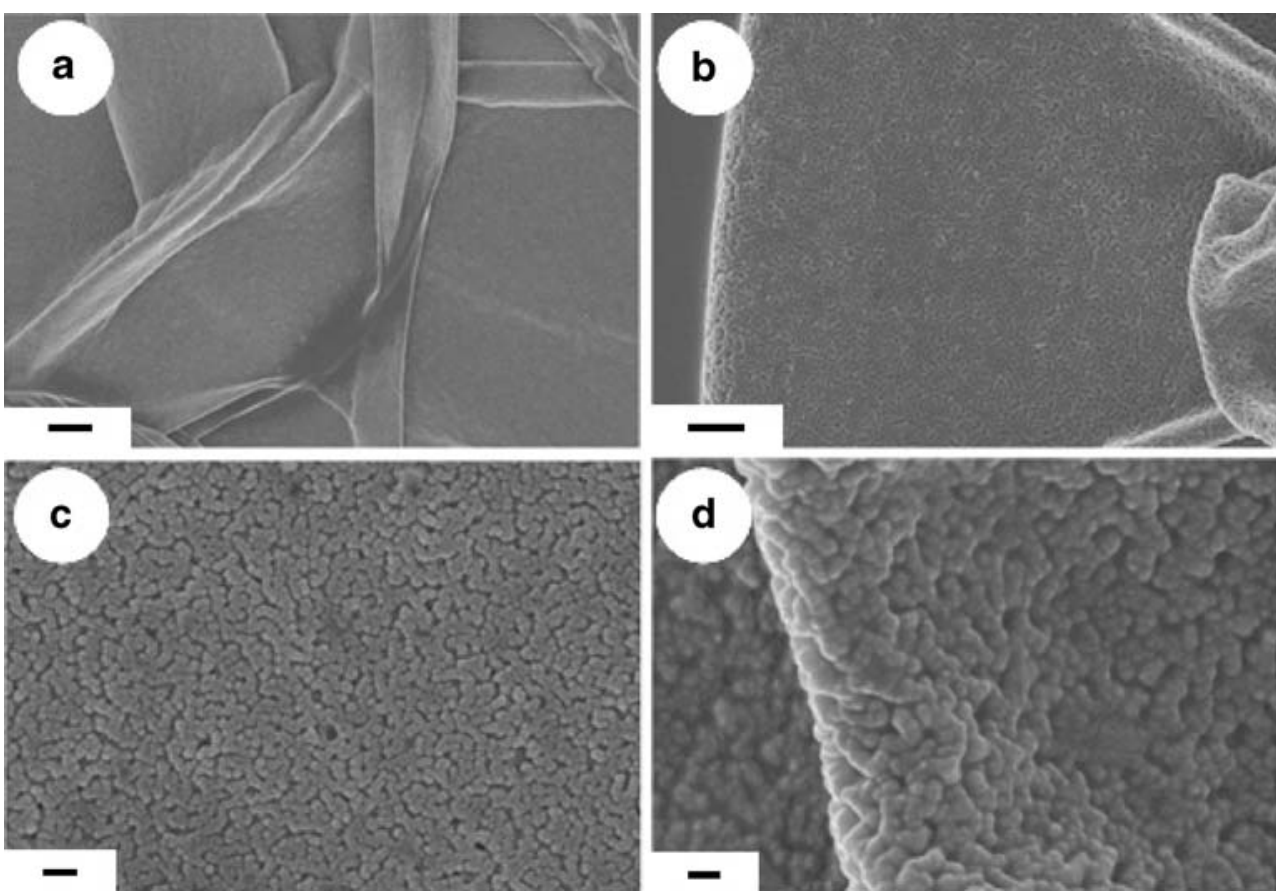

phase which depends on the initial concentration of the monomers [18].

The SEM images of Fig. 6 give an impression of the capsule size distribution.

The evaluation of the capsule size of the images of Fig. 6 suggests, if so ever, only little influence of the amount of shell polymer on the size. Considering the smallest and the largest capsules, the size range is 11-75, 19-74, 19-83, and 8-65 $\mu \mathrm{m}$ for panels $\mathrm{a}, \mathrm{b}, \mathrm{c}$, and $\mathrm{d}$, respectively. On the contrary, capsule morphology is considerably dependent on the amount of shell components for a given oil-to-water

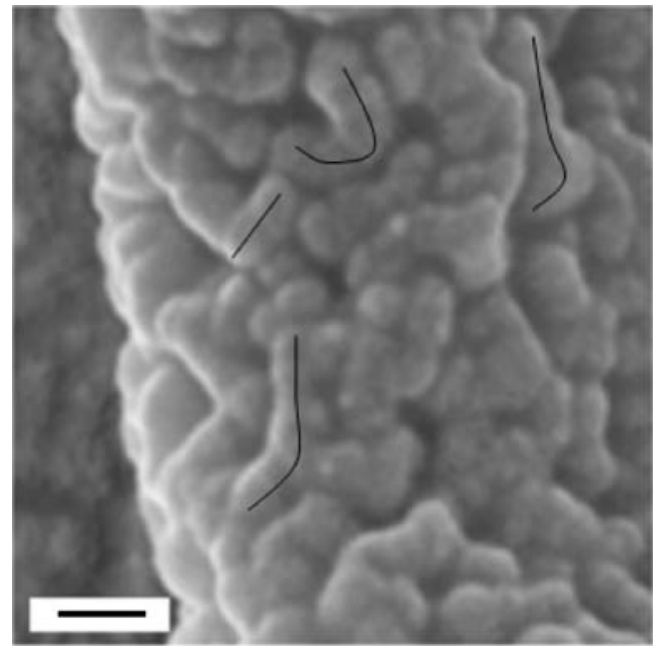

Fig. 5 Section of panel (d) of Fig. 4 showing string formation of polyamide particles and their behavior in a surface region of high curvature; the lines indicate individual strings; the bar indicates $100 \mathrm{~nm}$ ratio. Figure 7 exhibits individual capsules testifying the drastic changes with increasing amount of shell material.

The capsules with the lower amounts of shell polymer (panels a and b) collapse during drying almost completely, whereas the others (panels $\mathrm{c}$ and $\mathrm{d}$ ) appear much stronger, less collapsed, and retain partly their shape even in the vacuum of the SEM. The polymerization according to entry 4 of Table 1 (highest amount of shell components, panel d) led to capsules with quite strong shells that they appear in the SEM image almost like solid spheres with only shallow indentations and thin wrinkles.

Also, the morphology of the membrane on the nanometer scale changes with increasing amount of polyamide (Fig. 8). The membrane of the capsules with the lowest amount of shell material is porous with pore dimension in the order of up to $200 \mathrm{~nm}$ (Fig. 8a).

Increasing shell thickness brings about stepwise closing of the pores (cf. Fig. 8a-d). The capsule wall of the sample made with the highest amount of shell-forming monomers appears almost closed (Fig. 8d) as between the particles a smooth (film-like) polymer layer can be recognized. In other words, the particles appear as if they were embedded in a polymer film. The common morphological feature of all capsule membranes, despite the amount of shell polymer, is, however, the occurrence of strings as discussed above. Unfortunately, the SEM image taken with the highest magnification (Fig. 8d and insert) of the sample with the highest amount of shell material appears a little bit blurred; nevertheless, strings can also be recognized in this sample.

Another series of experiments has been carried out with varying content of cross-linker in the amine mixture (entries 
Fig. 6 SEM images displaying the capsule size distribution in dependence of the amount of both shell components while keeping the molar ratio between the acid dichloride (TPC) and the amine components (HMDA +DETA) unchanged (1:2); the amount of shell components varies in the order TPC/amine $0.5 / 1$ (entry 1 of Table $1, \mathbf{a}), 1 / 2$ (entry 2 of Table 1, b), 2/4 (entry 3 of Table 1, c), 3/6 (entry 4 of Table 1, d); the bar corresponds to $100 \mu \mathrm{m}(\mathbf{a}, \mathbf{b}, \mathbf{d})$ and $200 \mu \mathrm{m}(\mathbf{c})$
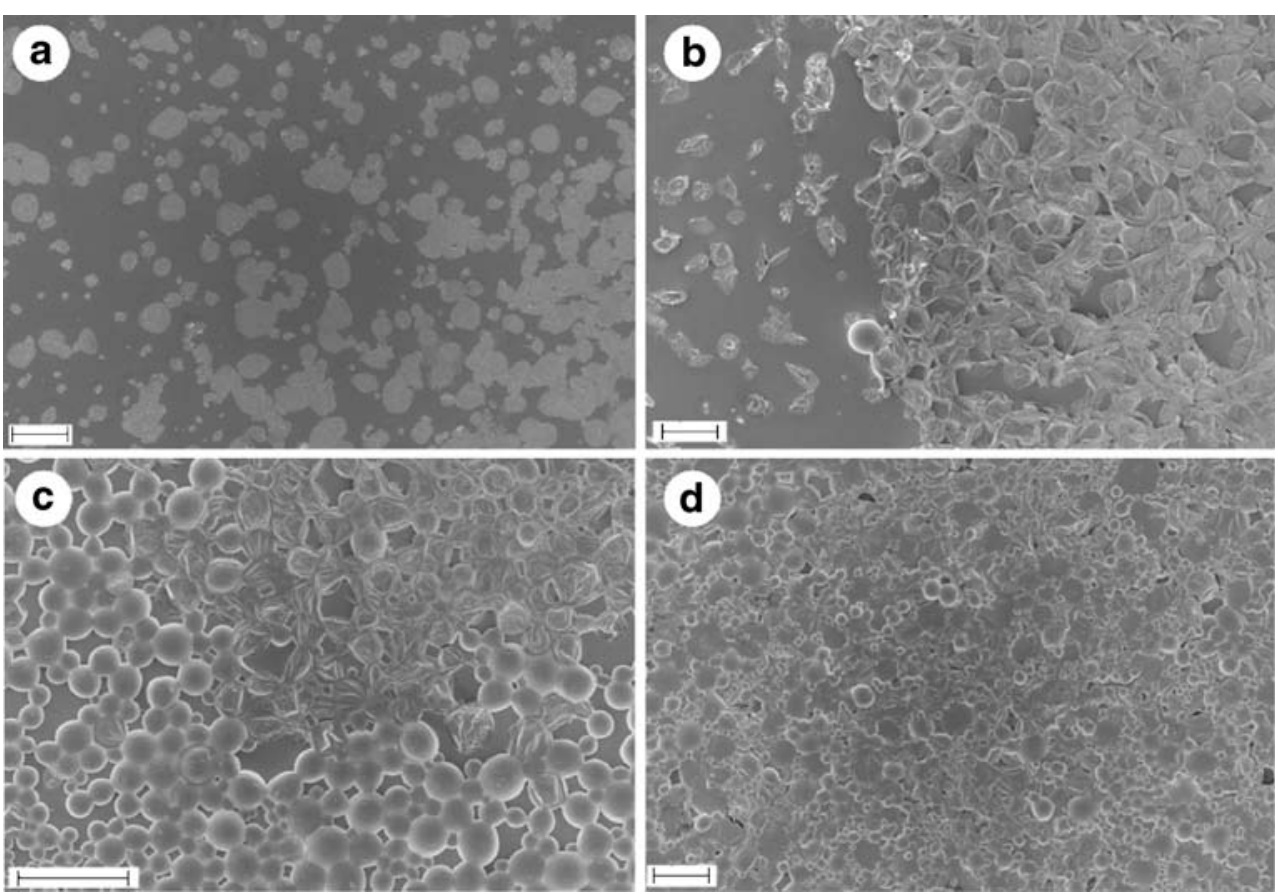

5 to 8 of Table 1), again keeping the acid dichloride to the overall amine ratio unchanged. Obviously, the formation of capsules does not depend whether or not the DETA crosslinker is present in the amine mixture as proven by the light microscopy images shown in Fig. 9. However, it is known that the presence of DETA cross-linker influences the mechanical properties as well as the porosity of the capsule wall [11, 13].

However, already light microscopy (Fig. 9) hints to possible differences regarding the capsule wall morphology and behavior during drying in dependence on the amount of DETA cross-linker in the amine mixture. The SEM images put together in Fig. 10 confirm this conclusion and, moreover, point to an effect on the capsule size. Evaluation of the size range of the capsules shows that it is between 26 and $133,21-100,41-92$, and $42-89 \mu \mathrm{m}$ for no, one fourth, three fourth, and only cross-linker in the amine mixture, respectively. Obviously, increasing the amount of crosslinker narrows the capsule size distribution and reduces the average size.
Fig. 7 SEM images of individual capsules with varying amounts of shell components while keeping the molar ratio between the acid dichloride (TPC) and the amine components (HMDA+ DETA) unchanged (1:2); the amount of shell components varies in the order TPC/amine $0.5 / 1$ (entry 1 of Table $1, \mathbf{a}), 1 / 2$ (entry 2 of Table 1, b), 2/4 (entry 3 of Table 1, c), 3/6 (entry 4 of Table 1, d); the bar corresponds to $2 \mu \mathrm{m}(\mathbf{a}, \mathbf{d})$ and $10 \mu \mathrm{m}(\mathbf{b}, \mathbf{c})$
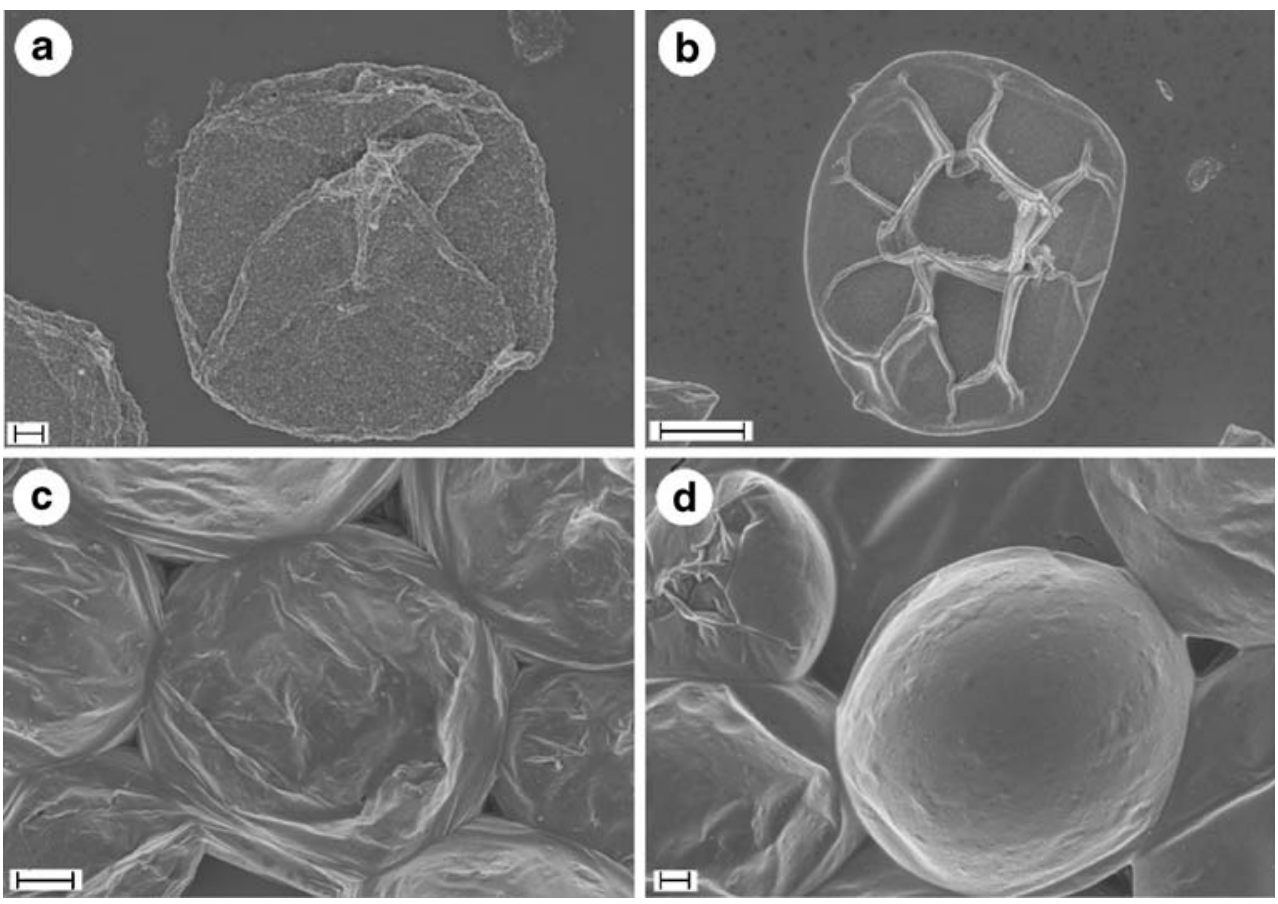
Fig. 8 Morphology of the capsules membrane in dependence on the amount of shell components while keeping the molar ratio between the acid dichloride (TPC) and the amine components (HMDA+DETA) unchanged (1:2); the amount of shell components varies in the order TPC/ amine $0.5 / 1$ (entry 1 of Table 1 , a), $1 / 2$ (entry 2 of Table $1, \mathbf{b}), 2 / 4$ (entry 3 of Table 1, c), 3/6 (entry 4 of Table 1, d); the bar corresponds to $200 \mathrm{~nm}(\mathbf{a}, \mathbf{b}, \mathbf{d})$ and $100 \mathrm{~nm}(\mathbf{c})$
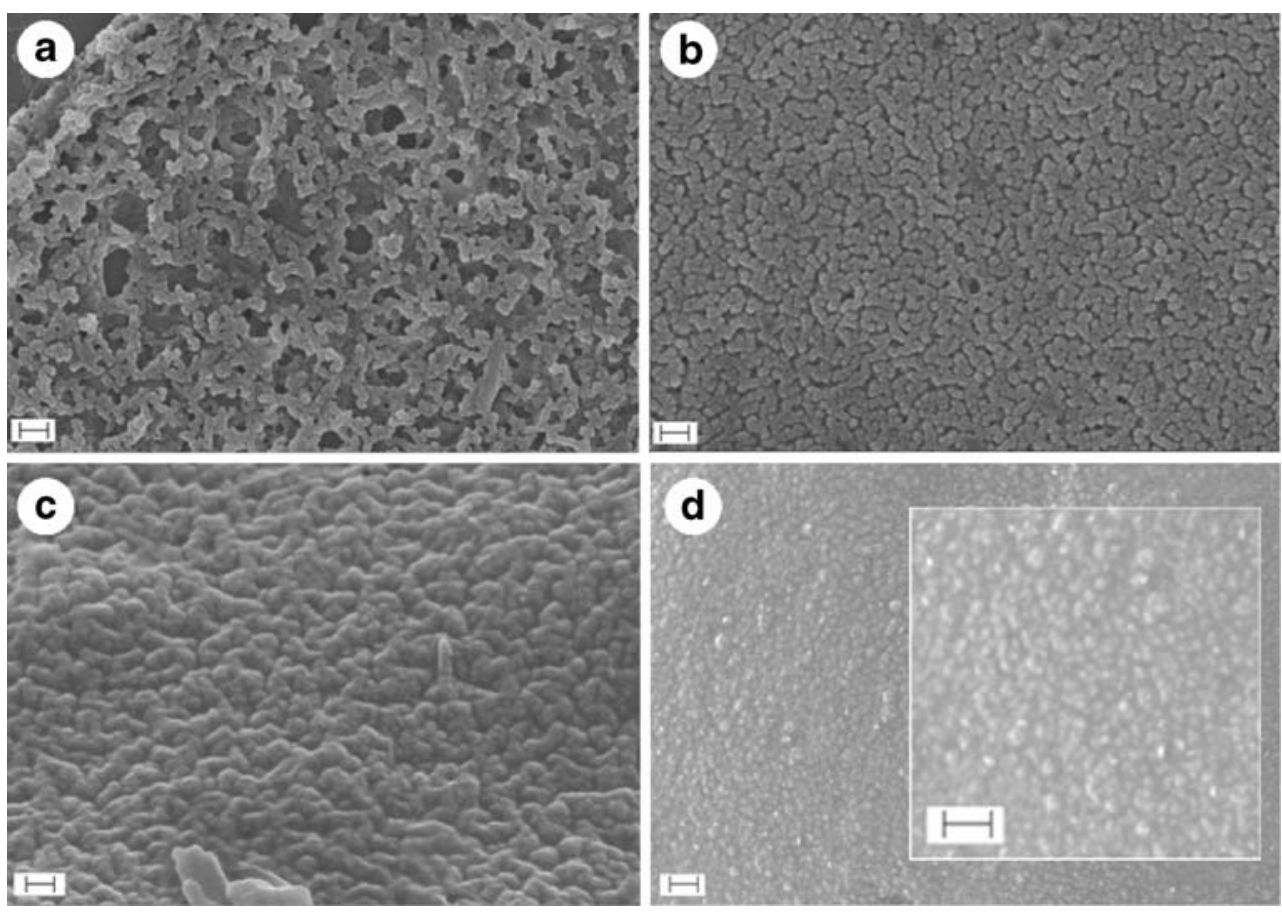

Zooming with the SEM into single capsules reveals how the cross-linker content influences the morphology of the capsule wall. The capsules of Fig. 11 illustrate that the appearance of the wrinkles changes quite drastically. With increasing amount of DETA, the wrinkles become thinner, their height reduces, and the thickness of the collapsed capsules also decreases. The reduction of the capsule height is proven by the thinner edge with increasing amount of cross-linker.

The flexibility of the capsule wall even in the presence of a cross-linker is impressively illustrated in Fig. 11b showing a capsule prepared with one third cross-linker in the amine mixture. Especially the central wrinkle performs several U-turns before it vanishes close to the capsule's edge on the left-hand side.

The content of DETA also influences the strings which, however, are present in all samples (cf. Fig. 12). Appar- ently, the length of the strings decreases with increasing amount of cross-linker in the amine mixture.

Reversing the composition of the oil phase, that is changing the cyclohexane-to-chloroform volume ratio from $4: 1$ to $1: 4$, should have an influence on the capsule morphology as at least the precipitation conditions are changing. How the capsule morphology on all hierarchical levels can be influenced is demonstrated with the examples given in Figs. 13 and 14. Note that Figs. 13 and 14 compare the influence of two experimental variables. First, the overall amount of monomers has been changed: it is twice as high for the capsules shown in Fig. 14 as for the capsules shown in Fig. 13. Second, the composition of the oil phase has been reversed: the cyclohexane-to-chloroform volume ratio is $4: 1$ for panels a and $\mathrm{c}$ and 1:4 for panels $\mathrm{b}$ and $\mathrm{d}$ in both figures.

Comparing panel $\mathrm{a}$ with $\mathrm{b}$ and $\mathrm{c}$ with $\mathrm{d}$ of both figures leads to similar conclusions independent of the total amount
Fig. 9 Light microscopy images of dried polyamide capsules prepared without cross-linker (a) and with 100\% cross-linker in the amine mixture (b) while keeping the molar ratio between the acid dichloride (TPC) and the amine components (HMDA+DETA) unchanged (1:2); the bar indicates $50 \mu \mathrm{m}$ in either case
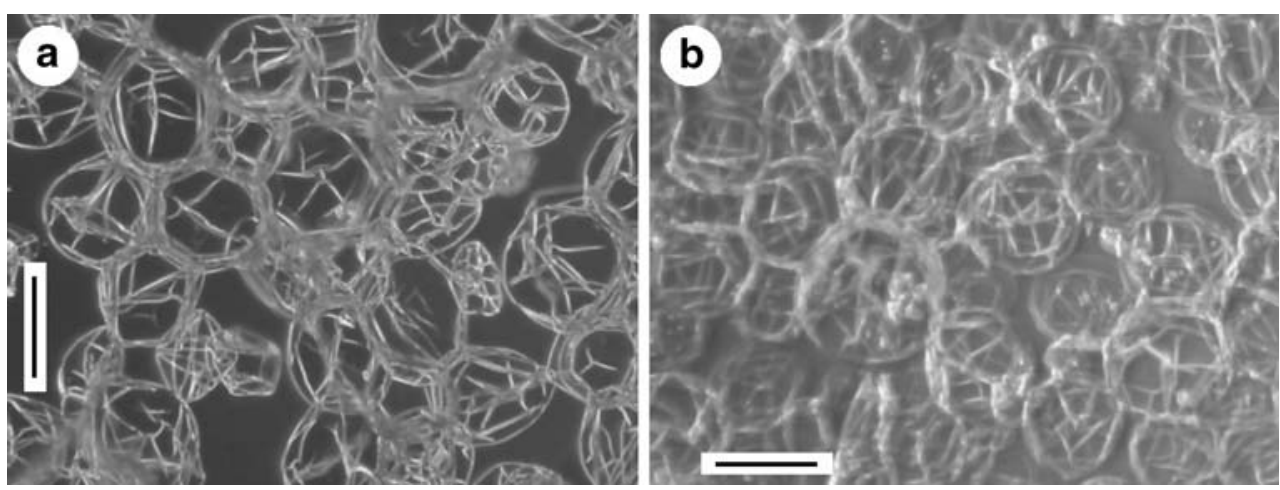
Fig. 10 SEM images displaying the capsule size distribution in dependence on the amount of DETA (cross-linker) in the amine mixture while keeping the molar ratio between the acid dichloride (TPC) and the amine components (HMDA+DETA) unchanged

(1:2); the amount of cross-linker varies in the order HMDA/DETA 2/0 (entry 5 of Table 1, a), 1.5/0.5 (entry 6 of Table $1, \mathbf{b}$ ), $0.5 / 1.5$ (entry 7 of Table $1, \mathbf{c}$ ), $0 / 2$ (entry 8 of Table $1, \mathbf{d}$ ); the bar corresponds to $200 \mu \mathrm{m}$ (a) and $100 \mu \mathrm{m}(\mathbf{b}, \mathbf{c}, \mathbf{d})$
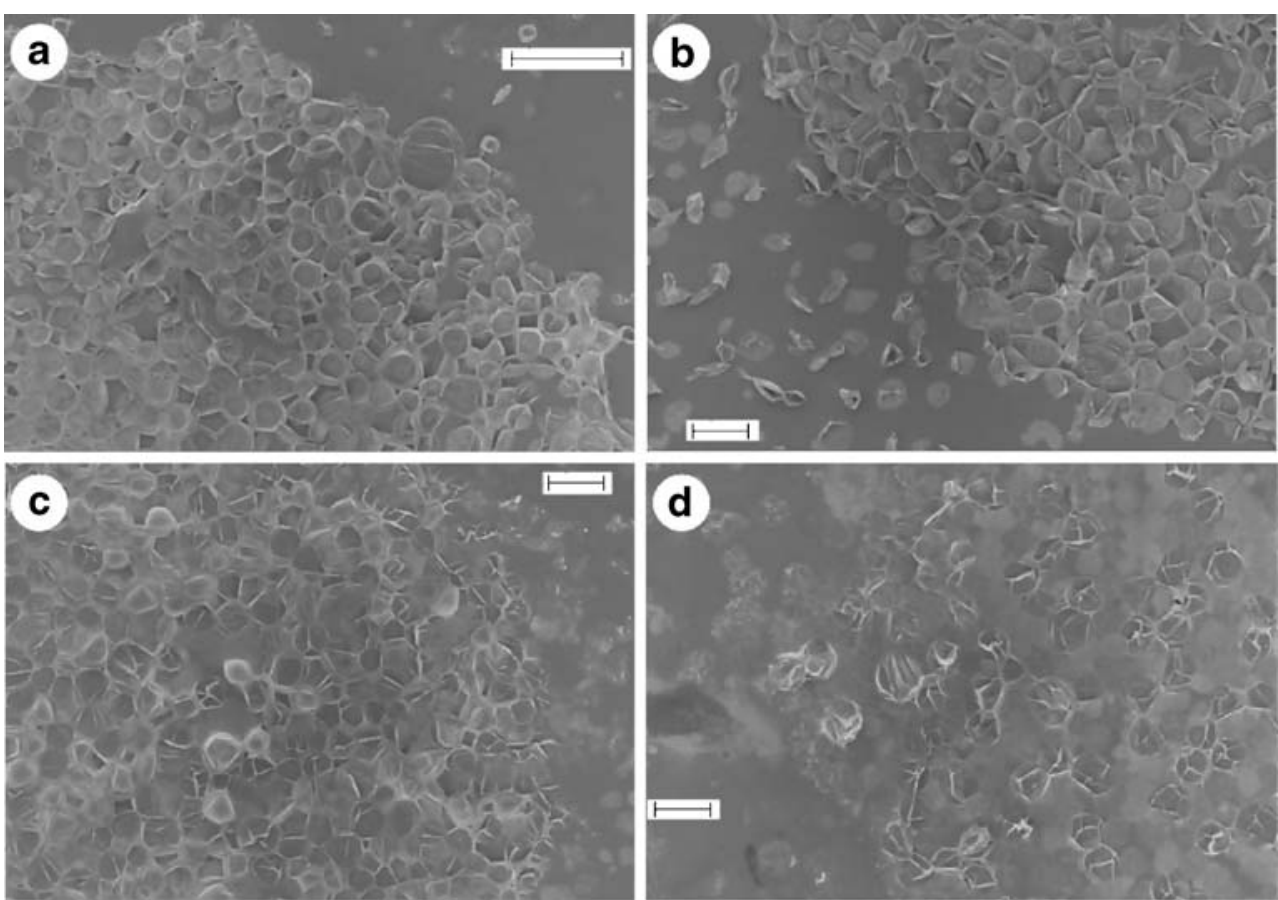

of shell components employed. This comparison shows that, with increasing amount of shell components, the thickness increases but the porosity of the membranes decreases (cf. also Figs. 6, 7, and 8 and discussion). Also, reversing the non-polar-to-polar oil ratio, which lowers the amount of cyclohexane, causes an increase in the thickness of the capsule wall. This result points to a strong influence of the polarity of the template oil phase on the capsule morphology. Moreover, with the higher chloroform content in the oil mixture, the capsules appear much cruder.

Panel $d$ of both Figs. 13 and 14 shows another interesting effect of the high chloroform content in the template oil. Besides the irregularly shaped pores, these SEM images reveal almost spherical openings much smaller in size than the surrounding polymer particles and resembling holes rather than pores (cf. Fig. 15). The
Fig. 11 SEM images displaying single capsules for varying amounts of DETA (cross-linker) in the amine mixture while keeping the molar ratio between the acid dichloride (TPC) and the amine components (HMDA+ DETA) unchanged (1:2); the amount of cross-linker varies in the order HMDA/DETA 2/0 (entry 5 of Table 1, a), 1.5/0.5 (entry 6 of Table 1, b), 0.5/1.5 (entry 7 of Table 1, c), 2/0 (entry 8 of Table 1, d); the bar corresponds to $2 \mu \mathrm{m}(\mathbf{a}, \mathbf{c})$ and $3 \mu \mathrm{m}(\mathbf{b}, \mathbf{d})$
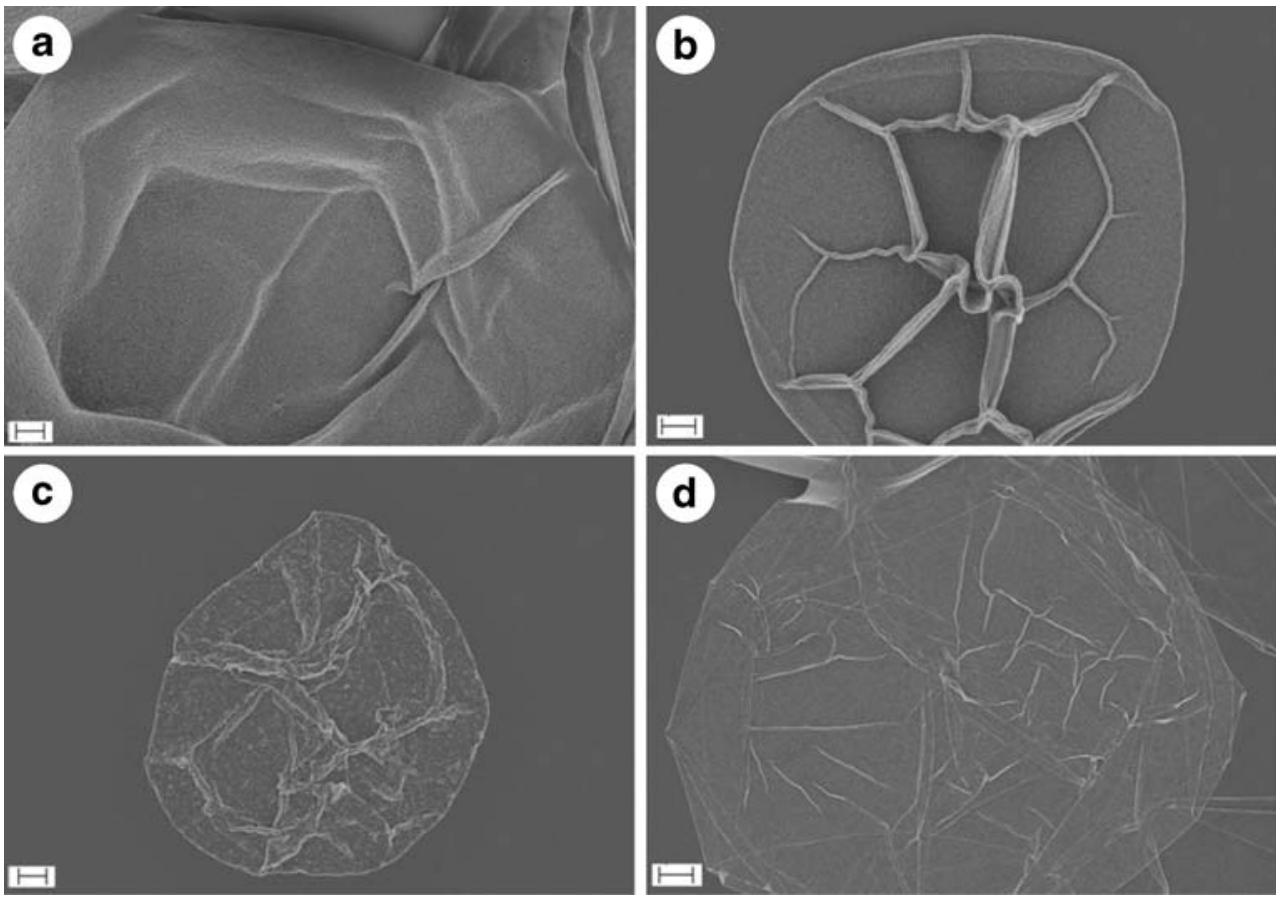
Fig. 12 SEM images of parts of the capsules' membrane in dependence on the amount of DETA (cross-linker) in the amine mixture while keeping the molar ratio between the acid dichloride (TPC) and the amine components (HMDA+DETA) unchanged (1:2); the amount of cross-linker varies in the order HMDA/DETA 2/0 (entry 5 of Table 1, a), 1.5/0.5 (entry 6 of Table $1, \mathbf{b}$ ), $0.5 / 1.5$ (entry 7 of Table $1, \mathbf{c}$ ), $2 / 0$ (entry 8 of Table $1, \mathbf{d}$ ); the bar indicates $200 \mathrm{~nm}(\mathbf{a}, \mathbf{c}, \mathbf{b}, \mathbf{d})$

Fig. 13 SEM images illustrating the capsules morphology with varying composition of the template oil phase. a, c

Cyclohexane-to-chloroform volume ratio $4: 1 ; \mathbf{b}, \mathbf{d}$ cyclohexaneto-chloroform volume ratio $1: 4$; the molar ratio between the acid dichloride (TPC) and the amine components (HMDA+DETA) is $0.5 / 1$; the bar indicates $20 \mu \mathrm{m}$ (a, b) and $200 \mathrm{~nm}(\mathbf{c}, \mathbf{d})$
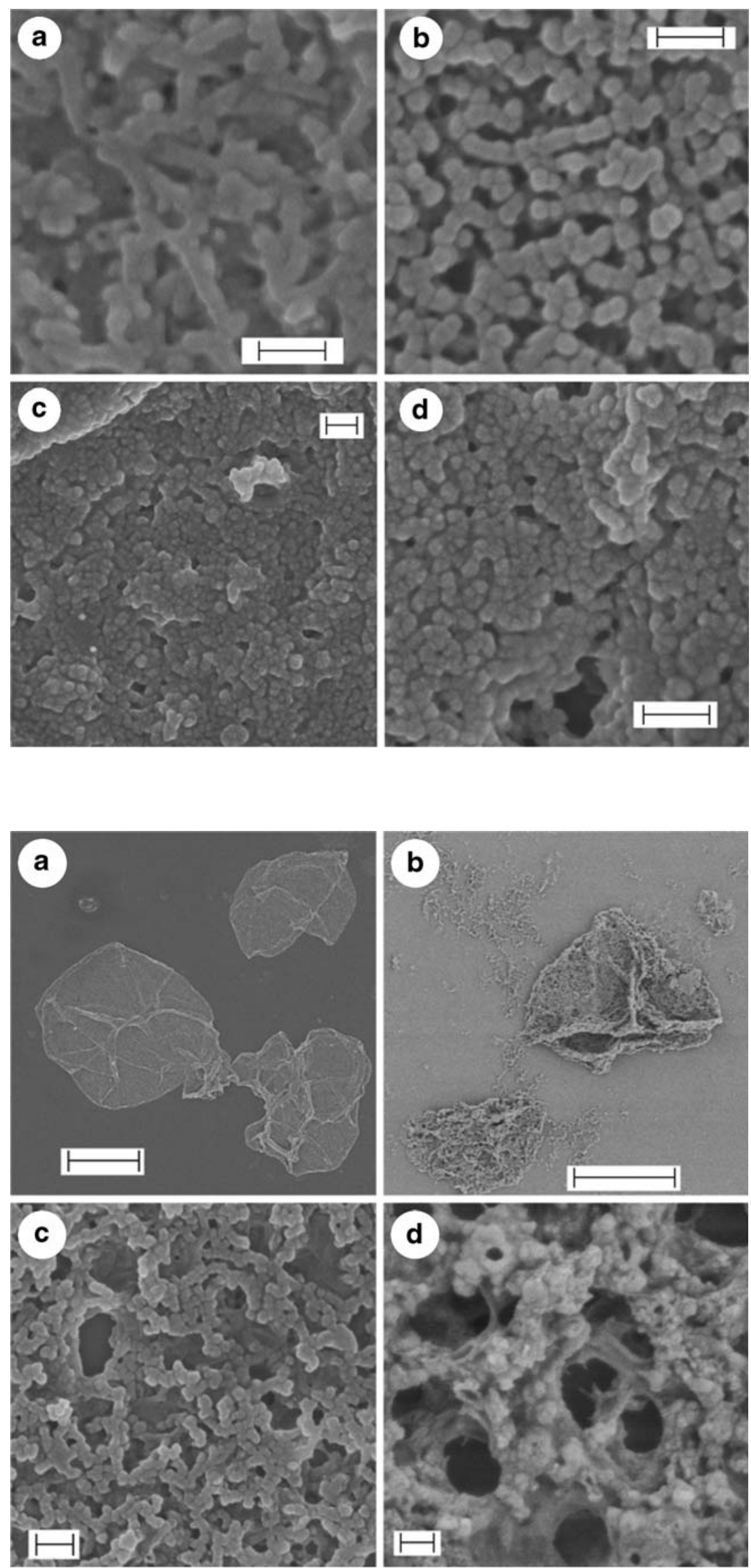
Fig. 14 SEM images illustrating the capsule morphology with varying composition of the template oil phase. a, c Cyclohexane-to-chloroform volume ratio $4: 1$; b, d cyclohexaneto-chloroform volume ratio $1: 4$; the molar ratio between the acid dichloride (TPC) and the amine components (HMDA + DETA) is $1 / 2$; the bar indicates $20 \mu \mathrm{m}$ (a, b), $200 \mathrm{~nm}(\mathbf{c})$, and $300 \mathrm{~nm}(\mathbf{d})$
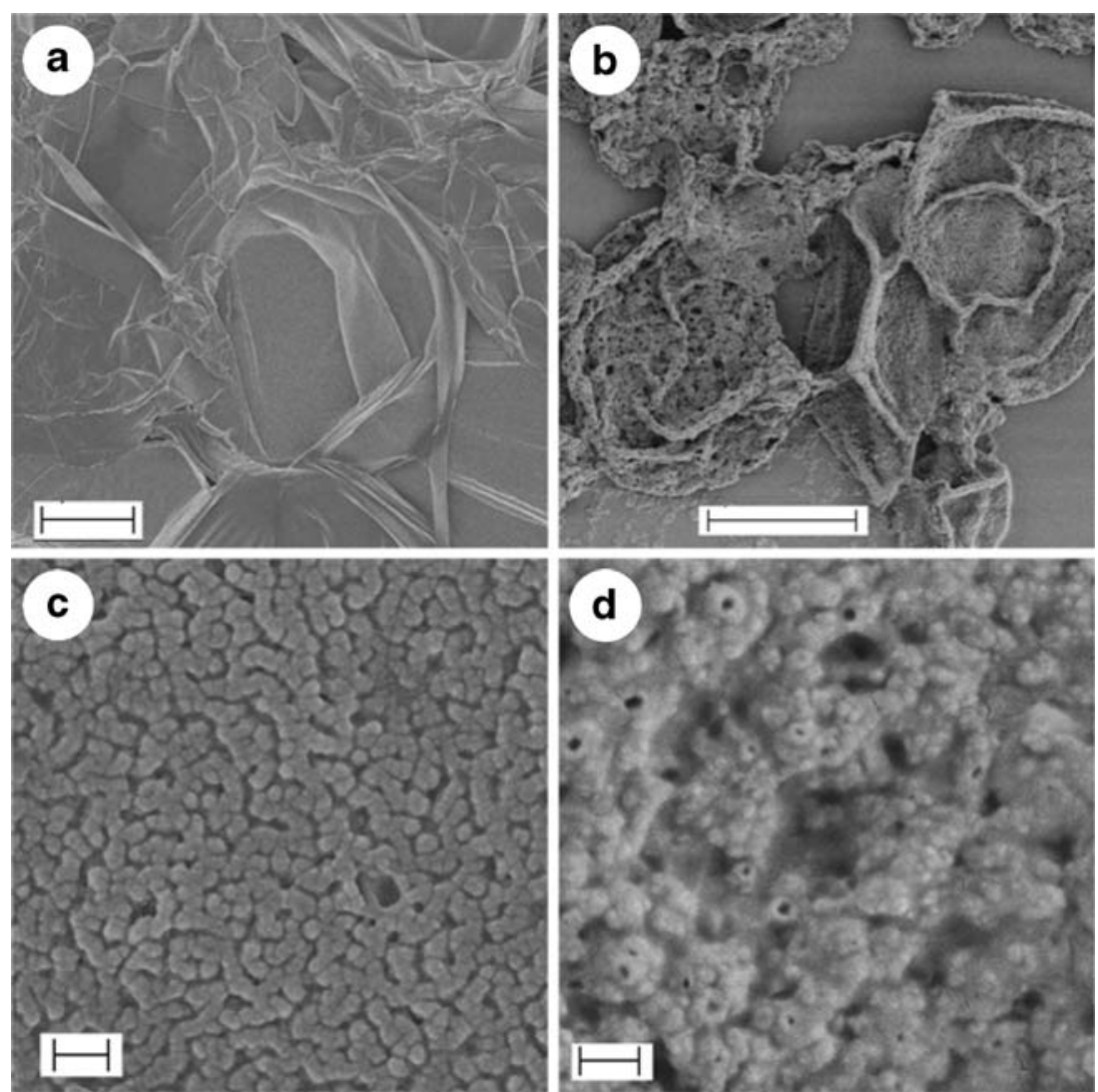

number of these holes increases greatly the higher the amount of shell components (cf. Figs. 13d and 14d). Figure 15 shows a part of the capsule membrane exhibiting a lot of the holes and illustrates that they possess quite a

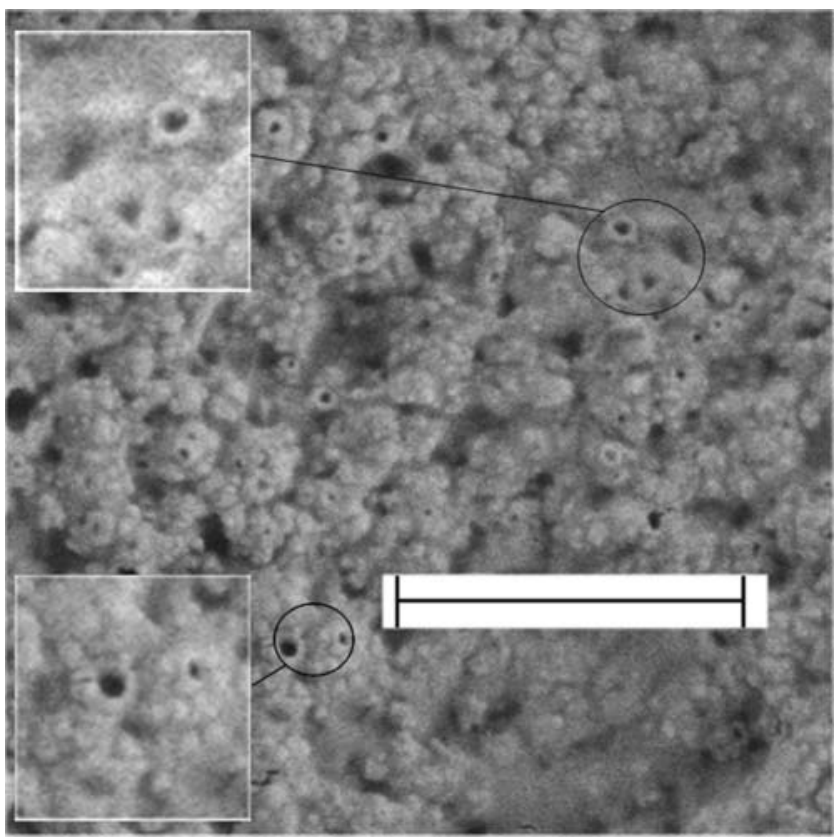

Fig. 15 Enlargements of panel (d) of Fig. 14 illustrating holes in the membrane of the capsules; the bar indicates $2 \mu \mathrm{m}$ broad size distribution. A possible explanation about the origin of these holes is discussed in the final chapter (Discussion and summary).

Polyamide-polystyrene composite capsules

The composition of the template oil phase can be modified in order to carry out specific further reactions. There are at least two general aims for such investigations. The first is to carry out chemical modifications of the membrane and the second is to gain better understanding of the particular reactions in more or less confined geometries. A simple approach to start with is radical polymerization of hydrophobic monomers such as styrene because this polymerization is fast and quite robust. Moreover, emulsion polymerization of styrene is the "standard" heterophase polymerization. Here, we report first results showing the proof of principle. A modification of the capsule wall might be possible via chain transfer to the polyamide which might be possible to the $\mathrm{CH}_{2}$ carbons.

The addition of styrene allows, after the polycondensation has finished, subsequent radical polymerization. Styrene is a non-solvent for polyamide and its presence in the template oil phase should not cause drastic changes of the morphology of the primary polyamide capsules. The SEM images of Fig. 16 suggest that if there is any effect, then it is only of minor importance as the general 
Fig. 16 SEM images of parts of the polyamide capsules' membrane in dependence on the amount of styrene in the oil phase (before polymerization of styrene); the overall volume of the oil phase is $15 \mathrm{ml}$; the volume ratio of oil phase to shell components and all other parameters correspond to entry 2 of Table 1; (a), (b), and (c) correspond to 0,2 , and $5 \mathrm{ml}$ of styrene, respectively; the bar indicates $200 \mathrm{~nm}(\mathbf{a}, \mathbf{c})$ and $300 \mathrm{~nm}(\mathbf{b})$
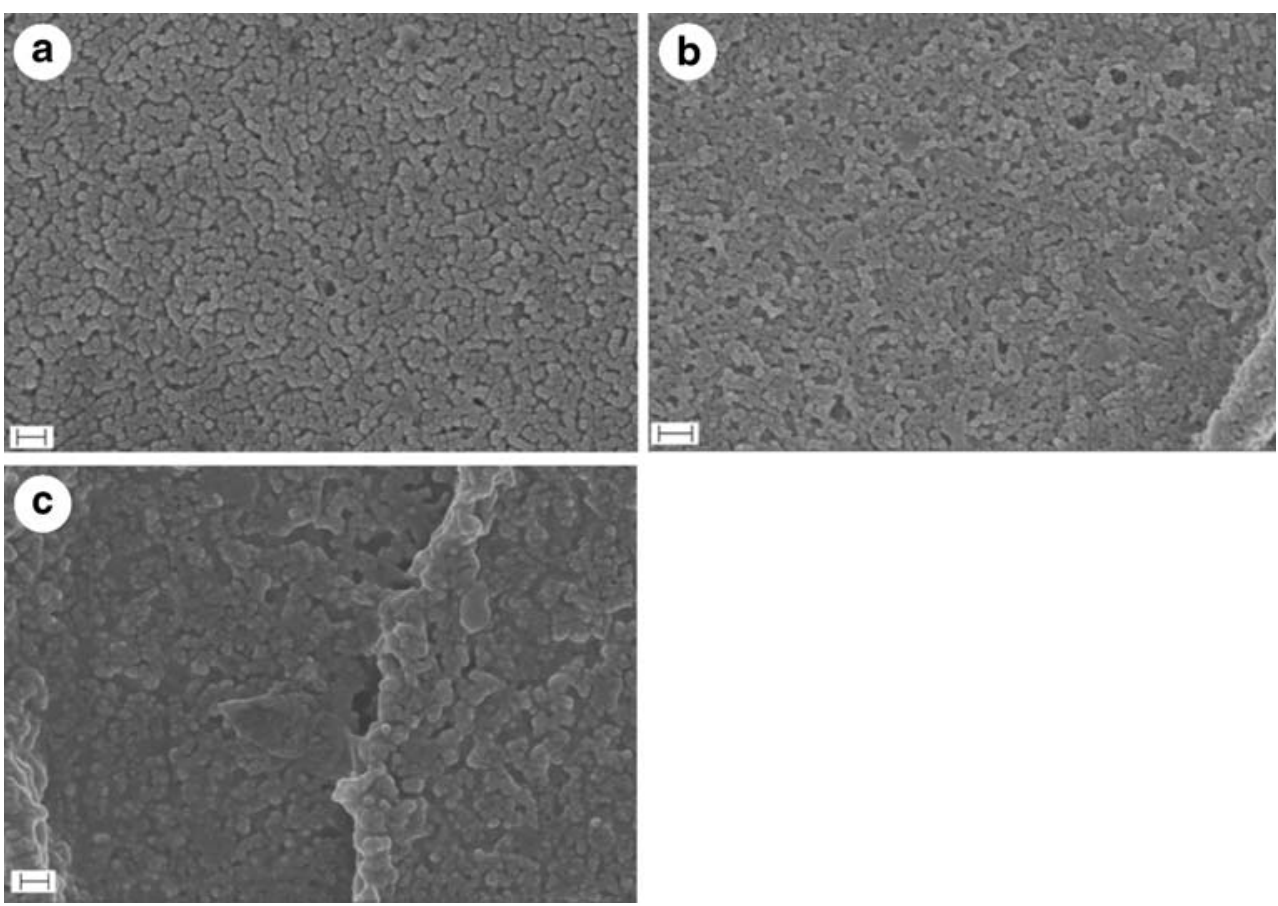

coagulation structure remains unchanged. However, the SEM images hint that with increasing volume fraction of styrene in the oil phase the precipitated polyamide particles appear increasingly blurred and connected by an interstitial polymer film. The morphology as depicted in Fig. 16c resembles those of Fig. $8 \mathrm{c}$ and $\mathrm{d}$ demonstrating the influence of increasing concentration of shell components in the oil phase.

In order to start the radical polymerization of styrene initiated with AIBN, the temperature was increased to $75^{\circ} \mathrm{C}$ after allowing the polycondensation to proceed for $1 \mathrm{~h}$ at room temperature. It is expected that this temperature jump has quite strong consequences regarding the partition of the components of the oil phase between the capsules and water. This involves cyclohexane, chloroform, AIBN (and its decomposition products), and styrene. Of course, the partition of AIBN and styrene might have consequences for the radical polymerization and for the eventual capsule morphology. As expected, the morphology of the final capsules drastically changes with increasing amounts of polystyrene (cf. Figs. 17 and 18).

Panels a and $\mathrm{c}$ of Figs. 17 and 18 show the typical features of the polyamide capsules with the possible effects of the changed composition of the oil phase by the styrene monomer. However, the final composite capsules show a different morphology (panels b and d of Figs. 17 and 18). Firstly, the composite capsules appear thicker compared with polyamide precursor (cf. panels a and b of Figs. 17 and 18). Secondly, the outer capsule membrane exhibits a completely different morphology that strongly depends on the amount of polystyrene (cf. panels c and d of Figs. 17 and 18). Polystyrene spheres are attached to the membrane. These polystyrene particles are only a few nanometers in size for the sample prepared with $2 \mathrm{ml}$ of styrene and only visible as tiny white spots (cf. insert of Fig. 17d) attached to the primary polyamide particles of the capsule wall. Increasing the styrene amount to $5 \mathrm{ml}$ leads to the formation of much larger polystyrene particles with a broad size distribution (from clearly below $50 \mathrm{~nm}$ up to about $500 \mathrm{~nm}$ ) and evidently recognizable attached to the outer capsule wall (cf. Fig. 18b and d).

The morphology of the composite particles suggests that the microcapsules do not only serve exclusively as microreactor for the styrene polymerization but also as template for the precipitation of latex particles born in the aqueous phase.

\section{Discussion and summary}

Polycondensation between terephthaloylchloride and a mixture (hexamethylenediamine/diethylenetriamine) takes place smoothly and reproducibly at the interface of aqueous emulsion droplets stabilized with poly(vinyl alcohol).

The oil drops act 2-fold. They serve as soft template and as storehouse for the acid dichloride. The polycondensation is started with the addition of the amine mixture to the aqueous phase.

The thickness and porosity of the capsule wall is controlled by the composition of the oil phase, the overall amount of the shell components (acid dichloride plus amine) in the reaction mixture, the relative amount of 
Fig. 17 SEM images of capsules and capsule membrane after finishing the polycondensation (a, c; polyamide capsule) and after styrene polymerization (b, d; composite capsule polyamide-polystyrene); the overall volume of the oil phase is $15 \mathrm{ml}$ including $2 \mathrm{ml}$ of styrene; the oil phase-to-shell components volume ratio and all other parameters correspond to entry 2 of Table 1; the bar indicates $10 \mu \mathrm{m}$ (a, b) and $200 \mathrm{~nm}(\mathbf{c}, \mathbf{d})$
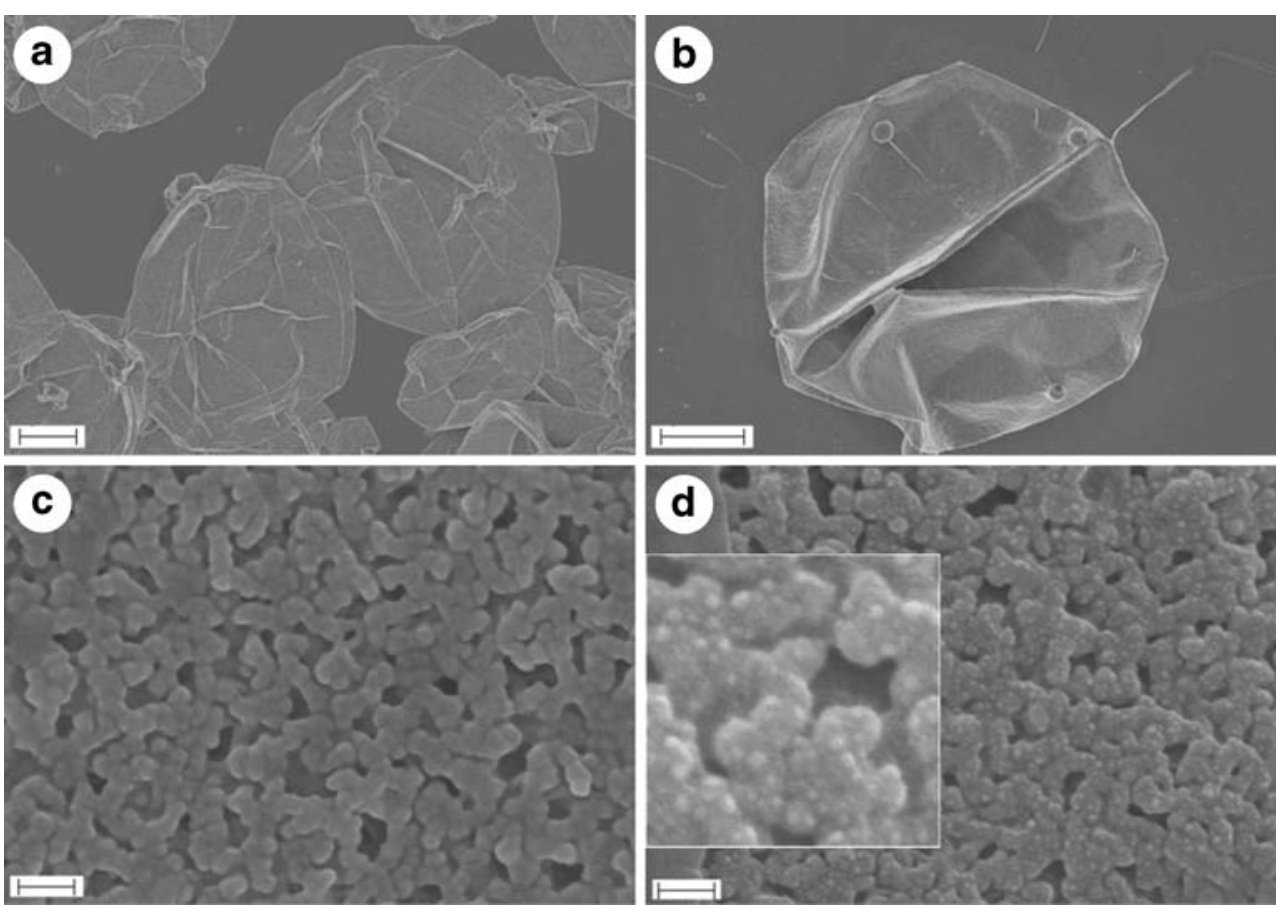

diethylenetriamine in the amine mixture, and whether a subsequent reaction is allowed after polycondensation.

The primary capsule wall consists of polyamide particles with a size below $50 \mathrm{~nm}$ adhering to the oil drops. These primary particles are arranged in strings made of up to about ten of them and, thus, they form an interfacial mesh or porous layer around the template oil drops with openings larger than their individual size. Through the pores, the oil phase can easily exchange with the environment. The primary polyamide particles adhere to the oil droplet-water interface as obviously the condition for complete engulfment by none of the fluid phases (either the oil or water) is met.

The primary polyamide particles are the results of precipitation polycondensation. Here, it should be noted that also in another templated precipitation polymerization string formation of primary particles has been observed as
Fig. 18 SEM images of capsules and capsule membrane after finishing the polycondensation (a, c; polyamide capsule) and after styrene polymerization (b, d; composite capsule polyamide-polystyrene); the overall volume of the oil phase is $15 \mathrm{ml}$ including $5 \mathrm{ml}$ of styrene; the oil phase-to-shell components volume ratio and all other parameters correspond to entry 2 of Table 1; the bar indicates $3 \mu \mathrm{m}$ (a, b) and $200 \mathrm{~nm}(\mathbf{c}, \mathbf{d})$
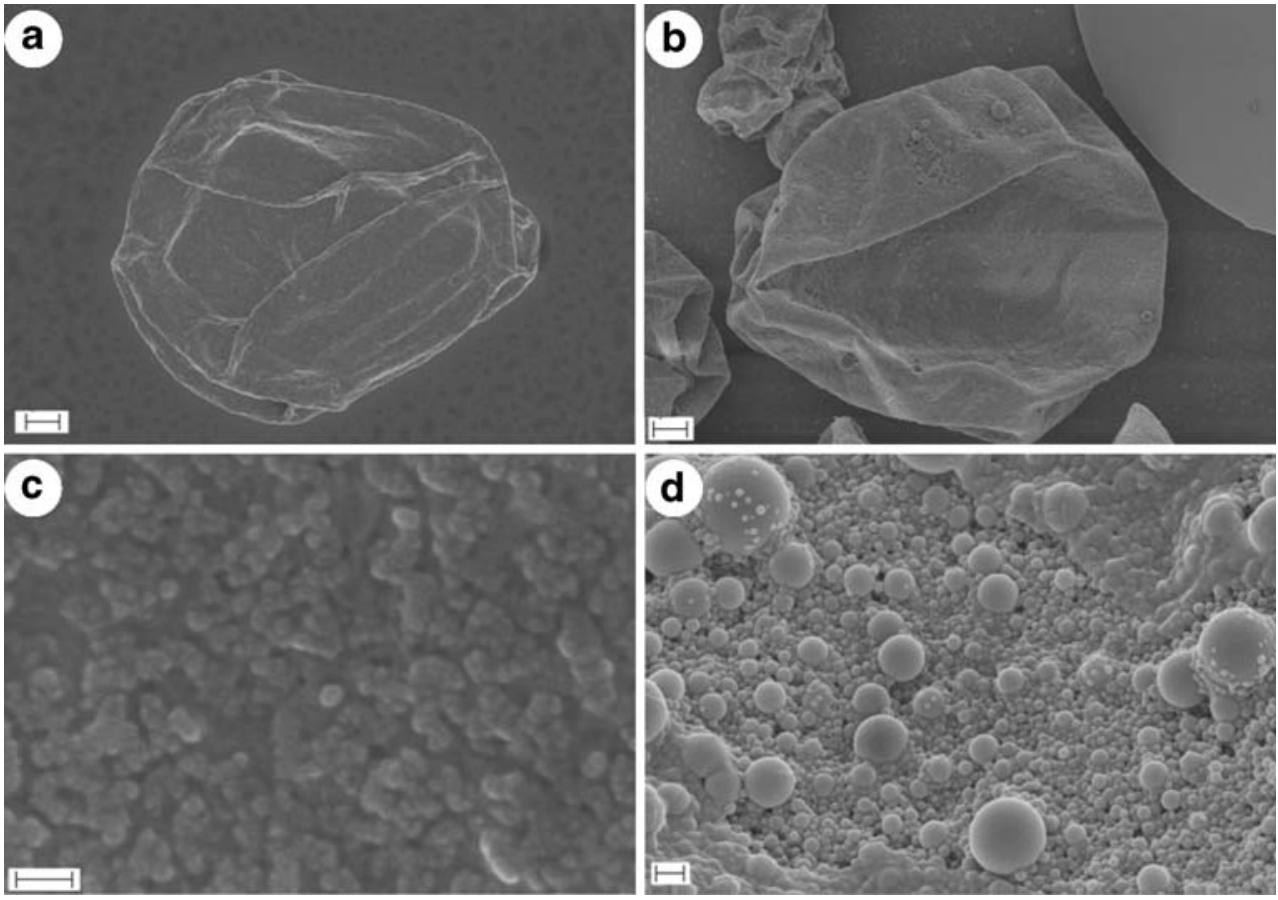
recently described for the oxidative polymerization of pyrrole $[29,30]$.

If the emptying capsules with thin membranes collapse, their surface is strewn with wrinkles. These wrinkles are regions of high curvature and their appearance illustrates the flexibility of the capsule wall. It is noteworthy to mention that the strings of primary particles do not restrict the flexibility of the capsule membrane. As also in the regions of high curvature the capsule wall is not broken, the conclusion can be drawn that the particles are interconnected by polyamide chains acting as movable joints between the primary particles as also discussed in the Introduction and in [2, 15-17]. This hypothesis is supported by the decreasing average length of the strings with increasing amount of cross-linker in the amine mixture (cf. Fig. 12).

An important aspect controlling the whole process at almost any stage and condition is the composition of the phases and the distribution as well as the state of all ingredients (not only the reactants!) between the phases. This conclusion is based on the following experimental facts and observations. Firstly, there is strong influence of the composition of the oil phase on the capsule morphology. Reversing the ratio between non-polar and polar oil has a dramatic effect on the morphology of the capsule wall. Secondly, polystyrene particles can be found at the outer side of the capsule membrane. Obviously, it is not only an interfacial region or inside the oil phase where the reactions take place but the continuous phase is important as well. The consideration of the following existing conditions gives a good base to understand the results. Firstly, thermodynamics drives to distribute all ingredients among all phases in order to bring differences of the chemical potentials to disappear (this holds for any species in any phase). Secondly, spontaneous emulsification of immiscible fluids in an interfacial layer causes a very special state of what is generally considered as solution. The former describes the general behavior of any reaction system on the way to minimize the free energy, whereas the latter allows a consistent explanation of the mentioned experimental facts. Spontaneous emulsification means that any contacting pair of immiscible fluids even in the absence of mechanical shear forces possesses the inherent tendency of droplet formation on either side of the interface [31]. The spontaneous droplet formation leads to quite broad size distributions containing everything from molecular aggregates (dimers, trimers, etc.) over nanometer-sized up to micrometer-sized droplets. If water is one of the liquids, the speed of droplet formation is the higher, the more polar the other liquid. For the reaction system considered here, this means that the higher the amount of chloroform in the oil phase, the faster and the more chloroform droplets (containing some cyclohexane and acid dichloride) are generated in the aqueous phase. Obviously, in this case, the interfacial condensation leads besides the capsules in the normal size range of a few tens of micrometers also to much smaller capsules. Upon drying, the small capsules form a single indentation rather than many wrinkles. These indentations are visible as holes on the SEM image of Figs. 14d and 15.

Also, styrene distributes between all phases and forms in the aqueous phase droplets which are crucial for the particle generation in the continuous phase even if the hydrophobic AIBN is used as thermal initiator [32,33]. Of course, with AIBN as initiator, the radical polymerization starts in both the capsules and the continuous aqueous phase. The polystyrene particles in the continuous phase were separated by centrifugation from the capsules. They possess an intensity-weighted average particle size (determined by dynamic light scattering) of 131 and $188 \mathrm{~nm}$ for the reactions with 2 and $5 \mathrm{ml}$ of styrene in the oil phase, respectively. Of course, there is a lower threshold regarding the amount of styrene that is necessary to get recognizable polymerization in the aqueous phase. For instance, an amount of only $0.5 \mathrm{ml}$ of styrene in the oil phase does not lead to latex formation but only to slightly modified capsules as discussed in context with Fig. 16. Obviously, the styrene polymerization simultaneously takes place inside the capsules and in the continuous water phase as emulsion polymerization. However, this conclusion needs further verification in future studies. Despite the need of further investigations, only this scenario, assuming two reaction sites, can explain, at least at the moment, the observed experimental facts of increasing capsule wall thickness and deposition of polystyrene particle on the outer capsule wall. Interestingly, the polystyrene particles of all sizes (cf. Figs. 17 and 18) stick quite tightly to the polyamide of the capsule wall as they could not be removed even by repeated centrifugation. Also, the composite capsules do sedimentate at the end of the polymerization (in contrast to the oil-filled polyamide capsules; cf. Fig. 1) due to the larger density caused by the attached polystyrene particles.

In conclusion, polyamide capsule formation via soft templating of oil drops shows all features of interfacial precipitation polymerization. The capsule wall consists of obviously interconnected polyamide particles. The composition of the oil phase strongly influences the thickness and the morphology of the capsule wall. Details of the capsule morphology and the results of second stage radical polymerization can be explained taking into account the distribution and the state of the components of the oil phase in the continuous water phase.

Acknowledgments The authors thank Mrs. Rona Pitschke and Mrs. Heike Runge for providing a huge number of SEM images. Thanks are also extended to Mrs. Ursula Lubahn and Mrs. Sylvia Pirok for technical assistance. HE gratefully acknowledges a GERSS (German Egyptian Research Short Scholarship) jointly funded by the Egyptian Ministry of Higher Education and Scientific Research (MHESR) and the German Academic Exchange Service (DAAD). The authors are also indebted to the Max Planck Society and the Max Planck Institute of Colloids and Interfaces for support of their research. 
Open Access This article is distributed under the terms of the Creative Commons Attribution Noncommercial License which permits any noncommercial use, distribution, and reproduction in any medium, provided the original author(s) and source are credited.

\section{References}

1. Blaiszik BJ, Caruso MM, McIlroy DA, Moore JS, White SR, Sottos NR (2009) Polymer 50:990

2. Mahabadi HK, Ng TH, Tan HS (1996) J Microencaps 13:559

3. Persico P, Carfagna C, Danicher L, Frere Y (2005) J Microencaps 22:471

4. Gan F, Yu C, Ma X, Zhao K (2008) Zhongguo Pige 37:28

5. Li J, Xue P, Han J, Sun G, Zhang M (2007) Jingxi Huagong 24:843

6. Regin AF, Solanki SC, Saini JS (2008) Renewable Sustainable Energy Rev 12:2438

7. Lapidot N, Gans O, Biagini F, Sosonkin L, Rottman C (2003) J Polym Sci 26:67

8. Caruso MM, Blaiszik BJ, White SR, Sottos NR, Moore JS (2008) Adv Funct Mat 18:1898

9. Caruso MM, Delafuente DA, Ho V, Sottos NR, Moore JS, White SR (2007) Macromolecules 40:8830

10. Arshady R (1989) J Microencaps 6:13

11. Mathiowitz E, Cohen MD (1989) J Membr Sci 40:1

12. Muramatsu N, Shiga K, Kondo T (1994) J Microencaps 11:171
13. Soto-Portas ML, Argillier JF, Mechin F, Zydowicz N (2003) Polymer Int 52:522

14. Toubeli A, Kiparissides C (1998) J Membr Sci 146:15

15. Mahabadi HK, Martin TI, Ng H, Patel R, Listigovers N, Yulo F (1990) Proc SPIE-Int Soc Opt Eng 1253:75

16. Tan HS, Ng TH, Mahabadi HK (1992) J Imag Sci Technol 36:587

17. Sundet SA (1993) J Membr Sci 76:175

18. Morgan PW, Kwolek SL (1959) J Polym Sci 40:299

19. Foti S, Maravigna P, Montaudo G (1983) Chim Industria 65:337

20. Zydowicz N, Chaumont P, Soto-Portas ML (2001) J Membr Sci 189:41

21. Alexandridou S, Kiparissides C, Fransaer J, Celis JP (1995) Surf Coat Technol 71:267

22. Enkelmann V, Wegner G (1972) Makromol Chem 157:303

23. Enkelmann V, Wegner G (1976) Makromol Chem 177:3177

24. Enkelmann V, Wegner G (1977) J Appl Polym Sci 21:997

25. Laguecir A, Louhaichi MR, Burgard M (2004) Curr Drug Deliv 1:111

26. Quevedo E, Steinbacher J, McQuade DT (2005) J Am Chem Soc 127:10498

27. Beaman RG, Morgan PW, Koller CR, Wittbecker EL, Magat EE (1959) J Polym Sci 40:329

28. Wittbecker EL, Morgan PW (1959) J Polym Sci 40:289

29. Diez I, Tauer K, Schulz B (2004) Coll Polym Sci 283:125

30. Diez I, Tauer K, Schulz B (2006) Coll Polym Sci 284:1431

31. Tauer K, Kozempel S, Rother G (2007) J Coll Interf Sci 312:432

32. Tauer K, Hernandez H, Kozempel S, Lazareva O, Nazaran P (2008) Coll Polym Sci 286:499

33. Tauer K, Mukhamedjanova M, Holtze C, Nazaran P, Lee J (2007) Macromol Symp 248:227 